\title{
A simplified formula to estimate the size of the cyclic plastic zone in metals containing elastic particles
}

\section{Andriollo, Tito; Kouznetsova, Varvara}

\section{Published in:}

Engineering Fracture Mechanics

Link to article, DOI:

10.1016/j.engfracmech.2020.107428

Publication date:

2021

Document Version

Peer reviewed version

Link back to DTU Orbit

\section{Citation (APA):}

Andriollo, T., \& Kouznetsova, V. (2021). A simplified formula to estimate the size of the cyclic plastic zone in metals containing elastic particles. Engineering Fracture Mechanics, 241, [107428].

https://doi.org/10.1016/j.engfracmech.2020.107428

\section{General rights}

Copyright and moral rights for the publications made accessible in the public portal are retained by the authors and/or other copyright owners and it is a condition of accessing publications that users recognise and abide by the legal requirements associated with these rights.

- Users may download and print one copy of any publication from the public portal for the purpose of private study or research.

- You may not further distribute the material or use it for any profit-making activity or commercial gain

- You may freely distribute the URL identifying the publication in the public portal 


\title{
A simplified formula to estimate the size of the cyclic plastic zone in metals containing elastic particles
}

\author{
Tito Andriollo $^{\text {a* }}$ and Varvara Kouznetsova ${ }^{\mathrm{b}}$ \\ ${ }^{\text {a }}$ Department of Mechanical Engineering, Technical University of Denmark, Denmark \\ ${ }^{\mathrm{b}}$ Department of Mechanical Engineering, Eindhoven University of Technology, The Netherlands \\ ${ }^{*}$ Corresponding author. Full postal address: Produktionstorvet, Building 425, room 214, 2800 Kgs. Lyngby, Denmark. \\ E-mail: $\underline{\text { titoan@mek.dtu.dk }}$
}

\begin{abstract}
The applicability of Irwin's estimate to metals containing particles becomes dubious when the plastic zone (PZ) size is comparable to the particle spacing. Therefore, a multiscale model is used to investigate the crack tip plasticity in a cyclically loaded elastic-perfectly plastic matrix containing a realistic distribution of spherical particles. Homogenization and dimensional analysis demonstrate that the PZ shape differs from what is expected based on von Mises plasticity. The scaling of the PZ size with the square of the stress intensity factor is confirmed, but the proportionality coefficient is strongly affected by the particle volume fraction. Consequently, a new compact formula for the PZ size is proposed, which holds for material properties ranging from metal matrix composites to cast irons and is robust to minor particle deviations from the spherical shape.
\end{abstract}

\section{Keywords}

Fatigue; Crack; Plastic Zone; Plastic Zone Size; Heterogeneous Materials; 


\section{Nomenclature}

\begin{tabular}{|c|c|}
\hline$a$ & Mean distance between centers of neighboring particles \\
\hline$a_{n n}$ & Particle center-to-center distance to the nearest neighbor \\
\hline$A$ & Geometrical parameter of the compact tension specimen \\
\hline$A_{p}$ & Area of the cyclic plastic zone \\
\hline$b$ & Thickness of the micro-scale resolved model \\
\hline$\underline{B}^{i, j}$ & $4 \times 4$ matrix containing the displacement and its derivatives at the corners of patch $(i, j)$ \\
\hline$B_{k m}^{i, j}$ & $(k, m)$ component of matrix $\underline{B}^{i, j}$ \\
\hline$d$ & Particle diameter \\
\hline$e_{i}$ & Cartesian unit vector along the $i$-axis \\
\hline$E$ & Young's modulus \\
\hline$f$ & Yield function \\
\hline$f_{V}$ & Particle volume fraction \\
\hline$F, F^{\prime}$ & Functions of the model parameters \\
\hline$h_{k}$ & Cubic Hermite interpolant \\
\hline$H_{k}$ & Kinematic hardening parameter \\
\hline$K_{I}$ & Mode I stress intensity factor \\
\hline$K_{\max }$ & Maximum value of $K_{I}$ over one cycle \\
\hline$l$ & Length of the Hermite interpolation interval \\
\hline$n_{x}, n_{z}$ & Number of patches along the $\mathrm{X}$ - and Z-axis \\
\hline$N$ & Mean number of particles per unit volume \\
\hline$P$ & Force per unit thickness applied to the loading pin \\
\hline$r_{p, f}$ & Forward plastic zone extent in the crack plane \\
\hline$r_{p, m}$ & Monotonic plastic zone extent in the crack plane \\
\hline$R$ & Load ratio \\
\hline$R_{p 02}$ & $0.2 \%$ proof stress \\
\hline $\mathbf{s}$ & Deviatoric part of the Cauchy stress tensor \\
\hline$s$ & Coordinate of the Hermite interpolant \\
\hline$S_{i, j}$ & Patch with indices $(i, j)$ \\
\hline$t$ & Surface traction vector \\
\hline $\boldsymbol{u}$ & Displacement vector \\
\hline$\widetilde{\boldsymbol{u}}$ & Interpolation of the displacement vector \\
\hline$u_{x}, u_{y}, u_{z}$ & Displacement components along the X-, Y- and Z-axis. \\
\hline$w_{c, p}$ & Normalized cyclic plastic work per unit volume \\
\hline $\bar{w}_{c, p}$ & Mean value of $w_{c, p}$ through the micro-scale model thickness \\
\hline$w_{p 02}$ & Plastic work per unit volume for monotonic loading up to $R_{p 02}$ \\
\hline$W$ & Geometrical parameter of the compact tension specimen \\
\hline$x$ & Position vector \\
\hline$x, y, z$ & Cartesian coordinates \\
\hline$x_{c t}$ & Crack tip coordinate along the $\mathrm{X}$-axis \\
\hline$\alpha$ & Back-stress tensor \\
\hline$\gamma_{k}$ & Kinematic hardening parameter \\
\hline
\end{tabular}




\begin{tabular}{|c|l|}
\hline$\delta_{b}$ & Thickness of the layer near the model boundaries \\
\hline$\varepsilon_{\mathbf{p}}$ & Plastic strain tensor \\
\hline $\bar{\varepsilon}$ & Effective uniaxial strain \\
\hline$\varepsilon_{i j}$ & Component $(i, j)$ of the strain tensor \\
\hline$\eta$ & Dimensionless threshold for plasticity detection \\
\hline$\lambda$ & Plastic multiplier \\
\hline$v$ & Poisson's ratio \\
\hline$\xi$ & Normalized coordinate of the Hermite interpolant \\
\hline$\Xi$ & Set of parameters characterizing the particle spatial distribution \\
\hline $\boldsymbol{\sigma}$ & Cauchy stress tensor \\
\hline $\bar{\sigma}$ & Effective uniaxial stress \\
\hline$\sigma_{y}$ & Yield stress \\
\hline$\psi$ & Dimensionless function approximating $\Psi$ \\
\hline$\Psi$ & Dimensionless function of the dimensionless model parameter groups \\
\hline$\partial \Omega^{Y-}, \partial \Omega^{Y+}$ & Micro-scale model face with normal pointing in the negative (resp. positive) Y-axis direction \\
\hline$[\quad]^{M}$ & Quantity refers to the macroscopic scale \\
\hline$[\quad]^{m}$ & Quantity refers to the matrix \\
\hline$[\quad]^{p}$ & Quantity refers to the particles \\
\hline $\mathrm{CSR}$ & Complete spatial randomness \\
\hline PZ & Plastic zone \\
\hline RVE & Representative volume element \\
\hline SGI & Spheroidal graphite iron \\
\hline
\end{tabular}

\section{Introduction}

The extent of the plastic zone at the crack tip is a parameter that plays a crucial role in the context of fatigue crack propagation. Indeed, the plastic zone corresponds to the region where irreversible processes take place and its size, relative to the characteristic microstructural dimension, determines the degree of microstructural sensitivity of the crack propagation process [1]. At the same time, the extent of the plastic zone is the key parameter to distinguish mechanically "long" from mechanically "short" cracks. Propagation of the former can be analyzed using linear elastic fracture mechanics concepts, whereas more advanced techniques are required to cope with the latter [2]. Reliable knowledge of the plastic zone size is thus essential both to understand the nature of the crack propagation as well as to select the most appropriate analysis approach.

Notable theoretical analyses of crack tip plasticity were conducted by Irwin [3] and Dugdale [4] in the early '60s and delivered closed-form approximations of the plastic zone size ahead of long cracks in homogeneous and isotropic non- 
hardening metals subjected to monotonic loading. For plane strain, the well-known expression proposed by Irwin reads

$$
r_{p, m} \approx \frac{1}{3 \pi}\left(\frac{K_{I}}{R_{p 02}}\right)^{2}
$$

where $r_{p, m}$ denotes the extent of the plastic zone in the direction along the crack, $K_{I}$ is the mode I stress intensity factor and $R_{p 02}$ is the $0.2 \%$ proof stress [5].

Under cyclic loading, which is the situation relevant to fatigue, the shape and extent of the plastic zone vary within each cycle [6]. In the published literature, two plastic zones are normally considered: the forward and reverse plastic zones, formed as the stress intensity factor approaches its maximum and minimum values, respectively. For constant amplitude cycling, load ratio $\mathrm{R}>0$ and plane strain, numerical analyses have shown that a reasonable estimate of the size of the forward plastic zone is

$$
r_{p, f} \approx 0.1\left(\frac{K_{\max }}{R_{p 02}}\right)^{2}
$$

where $K_{\max }$ is the maximum value of $K_{I}$ over one cycle and the factor 0.1 is taken as an average of the published data [7]. It is worth noting that since $0.1 \approx 1 / 3 \pi$, the formula above is essentially equivalent to the Irwin's estimate, provided that $K_{I}$ is replaced by $K_{\max }$.

While the applicability of the estimate (2) to most metallic materials is well accepted, its appropriateness becomes debatable for heterogeneous materials containing particles of the size comparable to the plastic zone size, e.g. metals containing elastic particles with dimensions in the range $1 \mu \mathrm{m}-1 \mathrm{~mm}$. These may be either artificially added to improve the mechanical properties, like in metal-matrix composites, or generated from the melt during the solidification process, like in cast irons. The concern around Eq. (2) stems from two factors. First, the homogeneity assumption breaks down when the plastic zone size is comparable to the particle spacing, which is not a rare situation for the two material classes mentioned above. Indeed, if a nodular cast iron with center-to-center particle spacing 100 $\mu \mathrm{m}$ and yield stress $250 \mathrm{MPa}$ is loaded at $\mathrm{R}=0.1$ near its fatigue threshold of $9 \mathrm{MPa} \cdot \mathrm{m}^{0.5}$, the plastic zone is expected to extend for $r_{p, f} \approx 160 \mu \mathrm{m}$. Second, there is experimental and numerical evidence that plasticity driven by the von Mises stress, which forms the backbone of the derivation of Eqs. (1) and (2), is not optimal to describe the inelastic deformation of metals containing particles $[8,9]$. 
Despite the dubious applicability of Eq. (2) to metals containing particles, only a few studies addressing this topic are available in the literature. Kassam et al. [10] pointed out the need to determine the elasto-plastic crack tip stress field in metal-matrix composites in the context of understanding fatigue life. They simulated the interaction between a crack and a cluster of particles and concluded that the particle spatial configuration can significantly affect the extent of plasticity in the nearby matrix. Miserez et al. [11] experimentally verified the scaling of the plastic zone size with $K_{I}^{2}$ during monotonic loading of Al-matrix composites, although they remarked that the definition of the elastic-plastic boundary in their study was somewhat arbitrary. Yi et al. [12] studied the interaction of a small crack with an elastic circular inclusion using a generalized Irwin approach, assuming monotonic loading. They concluded that the plastic zone size is smaller compared to the homogenous case when the inclusion is a rigid body, whereas it is larger when the inclusion is a void. A similar approach was used by Fan et al. [13] to investigate the behavior of a small ZenerStroh crack nucleated in the matrix of a fiber-reinforced composite. The study confirmed the primary role of the fiber stiffness relative to the matrix in controlling the plastic zone size. The fiber volume fraction was found to have an impact as well, although less pronounced than that of the fiber elastic stiffness. Concerning the shape of the plastic zone, Bouiadjra et al. [14] showed that the spatial distribution of the plastic strain at the tip of a crack in a homogeneous metal can differ significantly from that in a metal containing inclusions of the size comparable to the plastic zone. This finding was confirmed by Paul $[15,16]$, who conducted finite element analyses of a compact tension specimen under cyclic loading to determine the size and shape of the forward and reverse plastic zones in the presence of a limited number of soft and hard inclusions. In this regard, a vast body of literature is dedicated to investigating the impact of inclusions and voids on the fracture toughness of metals, e.g. [17-19]. Nevertheless, to the authors' best knowledge, most of these studies have a different focus and the features of the plastic zone have not been discussed in detail.

In summary, it appears that a systematic analysis of the applicability of Eq. (2) to metals containing particles has never been attempted. The present work aims at filling this gap by comparing the prediction of Eq. (2) to the output of a microstructure-resolved finite element model of a compact tension specimen. In contrast to the work of Paul [16] a large number of particles is considered here, sufficient to cover the full plastic region developing around the crack tip. A new formula to estimate the cyclic plastic zone size will be proposed to overcome the shortcomings of Eq. (2) that will be pinpointed throughout the analysis. 


\section{Material definition}

The material considered in this work is an idealized two-phase composite formed by an elastic-perfectly plastic metallic matrix containing a distribution of spherical elastic particles. The next sections describe the method used to generate the particles, the constituents' mechanical behavior and the homogenization procedure used to determine the effective properties of the composite.

\subsection{Microstructure}

A distribution of spherical particles was generated based on the microstructure of a nodular cast iron - also called spheroidal graphite iron (SGI) - similar to those considered by the authors in previous studies [20,21]. Following the procedure described in ref. [20], X-ray tomography was used to reconstruct the graphite morphology within a volume with dimensions 500x1600x1600 $\mu \mathrm{m}^{3}$. In total, 924 graphite nodules were identified, corresponding to a volume fraction $f_{V}=11.0 \%$. Next, each nodule was associated with a spherical particle having the same volume and centroid location. Figure 1 (a) shows the resulting size distribution, in terms of diameter $d$. It can be seen that the particles have diameters ranging from $15 \mu \mathrm{m}$ to $140 \mu \mathrm{m}$, with an average value of $51 \mu \mathrm{m}$. To characterize their spatial distribution, the center-to-center distance to the nearest neighbor $a_{n n}$ was computed for each particle. Figure 1 (b) compares the computed cumulative distribution function of $a_{n n}$ with the theoretical expectation based on the hypothesis of complete spatial randomness (CSR) [22]. The comparison reveals that the values of $a_{n n}$ for the present set of particles are somewhat larger than what would be expected according to the CSR hypothesis. The discrepancy cannot be attributed to edge effects, as the situation does not change by discarding the values of $a_{n n}$ associated with the particles near the

volume boundaries. Qualitatively, values of $a_{n n}$ smaller than under CSR indicate clustering, whereas the opposite indicates regularity [22]. Therefore, the data of Figure 1 (b) suggest that the present particles are rather regularly spaced instead of randomly distributed, which is not surprising in light of the mechanism by which the graphite nodules form [23]. 


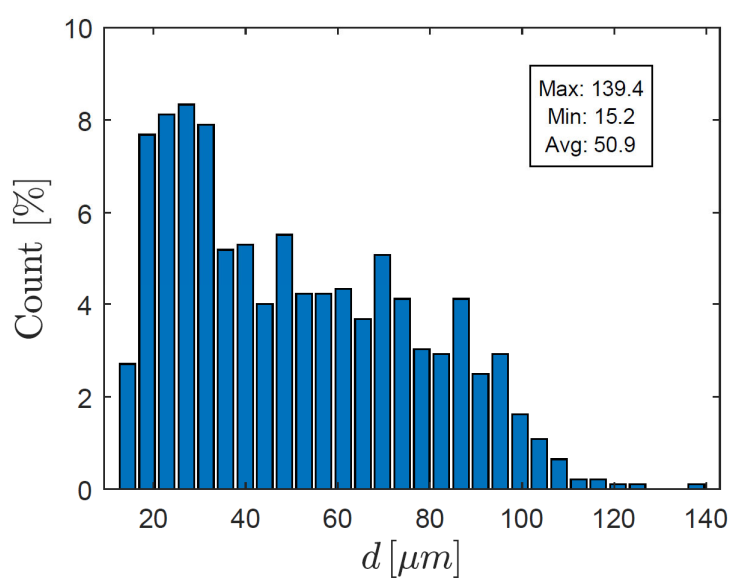

(a)

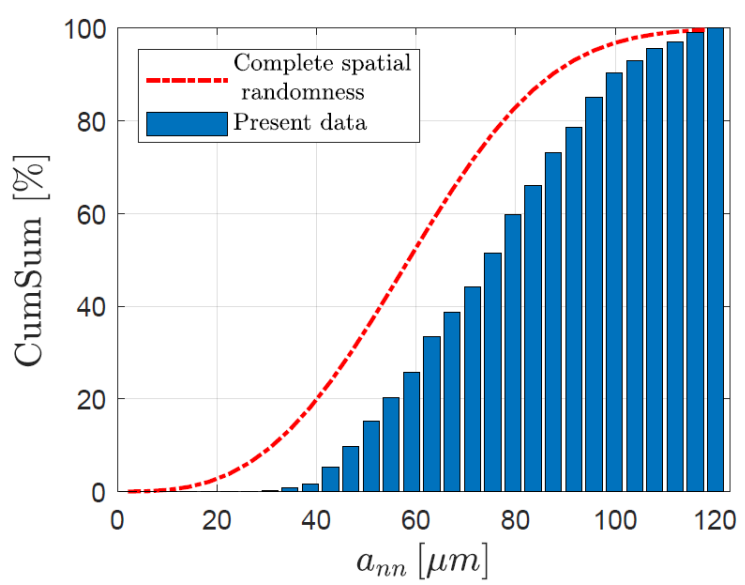

(b)

Figure 1 - (a) Distribution of the generated particle diameter. (b) Cumulative distribution of the generated particle center-to-center distance to the nearest neighbor.

\subsection{Constituent material models}

The constituent material models were selected based on the behavior of ductile metals containing elastic-brittle particles with minimum particle size greater than 1 micron, e.g. common metal-matrix composites and cast irons. In addition, simple models were preferred over more involved ones in order to keep the overall number of parameters as low as possible and therefore simplify the sensitivity analysis that will be presented in Section 4.3. Accordingly, the matrix was modelled as isotropic linear elastic-perfectly plastic, with the flow behavior described by the standard J2 (von Mises) plasticity theory. The spherical particles were assumed isotropic linear elastic with an infinite bond strength of the particle-matrix interface.

Two sets of elastic and plastic parameters were considered, representative of the behavior of SGI [24] and Al reinforced with $\mathrm{SiC}$ [25]. For both sets, the parameter values are reported in Table 1 , where $E^{m}$ and $v^{m}$ denote the Young's modulus and Poisson's ratio of the matrix, respectively, $E^{p}$ and $v^{p}$ denote the same quantities for the particles and $\sigma_{y}^{m}$ is the matrix yield stress. As this latter quantity can vary significantly within both the SGI and Al$\mathrm{SiC}$ families, representative average values are reported in Table 1. In the subsequent analysis, however, the matrix yield stress will be varied during parameter sensitivity studies to cover the full range of realistic values. 
Table 1 - Assumed elastic and plastic parameters of the matrix and the particles.

\begin{tabular}{cccccc}
\hline & $E^{m}[\mathrm{GPa}]$ & $v^{m}$ & $E^{p}[\mathrm{GPa}]$ & $v^{p}$ & $\sigma_{y}^{m}[\mathrm{MPa}]$ \\
\hline $\mathrm{SGI}$ & 205.0 & 0.290 & 15.0 & 0.3 & 500.0 \\
$\mathrm{Al}-\mathrm{SiC}$ & 75.0 & 0.330 & 410.0 & 0.2 & 200.0 \\
\hline
\end{tabular}

\subsection{Effective behavior}

Knowledge of the homogenized (effective) behavior of the composite is needed for two reasons: to estimate the size of its cyclic plastic zone by means of Eq. (2) and to simulate the material elastic response far from the crack tip in the model described in Section 0. The effective behavior was determined by subjecting a Representative Volume Element (RVE) of the composite microstructure to virtual uniaxial testing. The RVE, shown in figure 2 (a), corresponds to a $450 \times 450 \times 450 \mu \mathrm{m}^{3}$ sub-region of the volume considered in Section 2.1 and contains 74 spherical particles.

A geometrically linear finite element model of the RVE was created in ABAQUS and meshed with $\approx 300000$ 4-node hybrid displacement-pressure elements, using a minimum element size of $\approx 10 \mu \mathrm{m}$. Periodic boundary conditions were imposed on the non-periodic mesh of the model following the approach of Nguyen et al. [26], using Lagrange polynomials of degree 9 . The macroscopic effective uniaxial stress $\bar{\sigma}$ and strain $\bar{\varepsilon}$ were determined as the volume averages of the corresponding microscopic local quantities using the simplified formulas reported in ref. [27]. Negligible differences in terms of the computed effective properties were found by comparing the results of uniaxial tests simulated along different directions, indicating the isotropy of the effective RVE response. In addition, variations below $0.5 \%$ were detected by halving the mesh element size. Therefore, both the RVE and the mesh size were considered appropriate.

Figure 2 (b) shows a few uniaxial tension-compression curves computed with the RVE model using SGI material properties (see Table 1). The reported data correspond to five loading cycles at prescribed strain amplitude of $0.5 \%$, $1.0 \%, 3.0 \%$ and $5.0 \%$. It is evident that a stabilization of the RVE response is achieved already after the first loading cycle. Furthermore, the cycles are symmetric with respect to the origin, i.e. the uniaxial responses in tension and in compression coincide, indicating negligible influence of the residual stresses. The same conclusions hold for the Al$\mathrm{SiC}$ material parameters.

The effective parameters of the composite were determined by fitting the uniaxial response of the RVE model. The values are listed in Table 2, where $E, v$ and $R_{p 02}$ are the Young's modulus, Poisson's ratio and $0.2 \%$ proof stress, 
respectively; $w_{p 02}$ is the computed plastic work per unit volume for the monotonic loading up to $R_{p 02}$. The reported values of the effective Young's modulus and proof stress of Al-SiC and the effective Young's modulus of SGI compare well with the measured values $[25,28]$. By contrast, the effective proof stress of SGI appears overestimated by 5 to $10 \%$ [29]. A possible reason is the strong matrix-particle interface assumed in the model, which does not allow accounting for the gradual particle-matrix debonding that takes place in reality during actual tensile loading of SGI [20].

Table 2 - Effective parameters of the composite.

\begin{tabular}{ccccc}
\hline & $E[\mathrm{GPa}]$ & $v$ & $R_{p 02}[\mathrm{MPa}]$ & $w_{p 02}[\mathrm{MPa}]$ \\
\hline SGI & 172.2 & 0.280 & 443.0 & 0.858 \\
Al-SiC & 90.9 & 0.314 & 231.3 & 0.445 \\
\hline
\end{tabular}

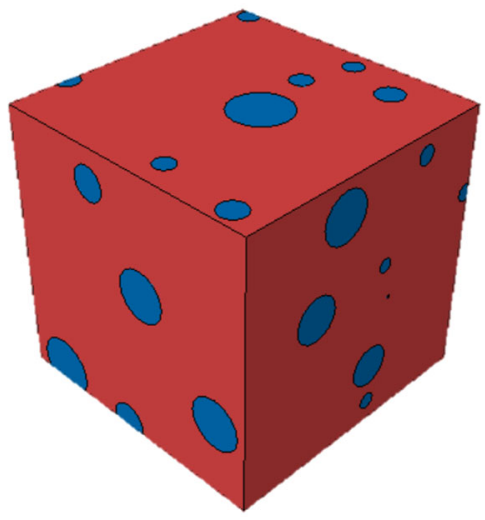

(a)

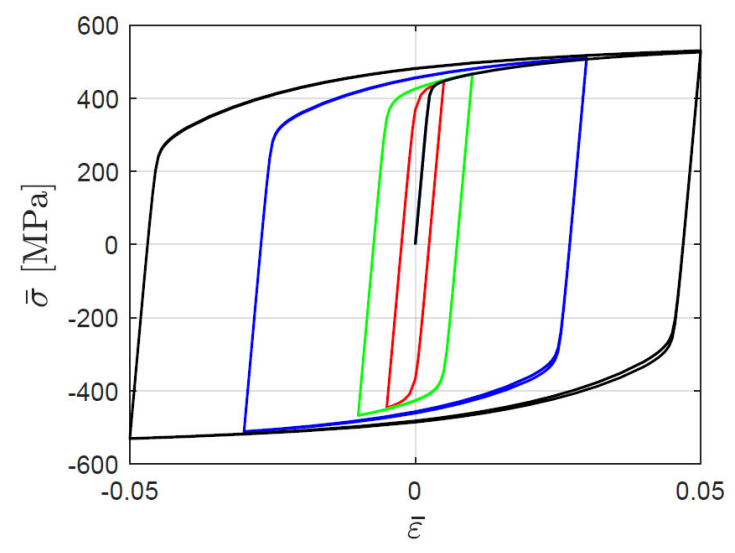

(b)

Figure 2 - (a) Representative volume element (RVE) used to determine the effective mechanical behavior of the composite. (b) Simulated uniaxial tension-compression curves for prescribed strain amplitudes of $0.5 \%$ (red), $1.0 \%$ (green), 3.0\% (blue) and 5.0\% (black). The data correspond to 5 loading cycles and were obtained using the RVE with the material properties of SGI, see Table 1. 


\section{Model of the compact tension specimen}

A multiscale approach was used to investigate the cyclic plastic zone in a virtual compact tension specimen made of the composite material described in the previous section. The approach is based on two finite element models (see figure 3): a 2D macro-scale model that represents the entire specimen and a 3D micro-scale resolved model that is limited to the region surrounding the crack tip. The purpose of the macro-scale model is to determine the displacement field at a sufficient distance from the crack tip, assuming the material homogeneous. This displacement field is then used to prescribe the boundary conditions on the micro-scale model, where the microstructure is fully resolved.

It is worth noting that the need for the macroscale model could be avoided by imposing the standard K-field solution directly on the boundary of the micro-scale model [5]. This would be equivalent to assuming that the boundary is located within the K-field dominated zone. However, this assumption would be hard to fulfill over a range of different loads and material properties without changing the geometry of the micro-scale model. Therefore, the multiscale approach was deemed more appropriate in the present case.

2D macro-scale model

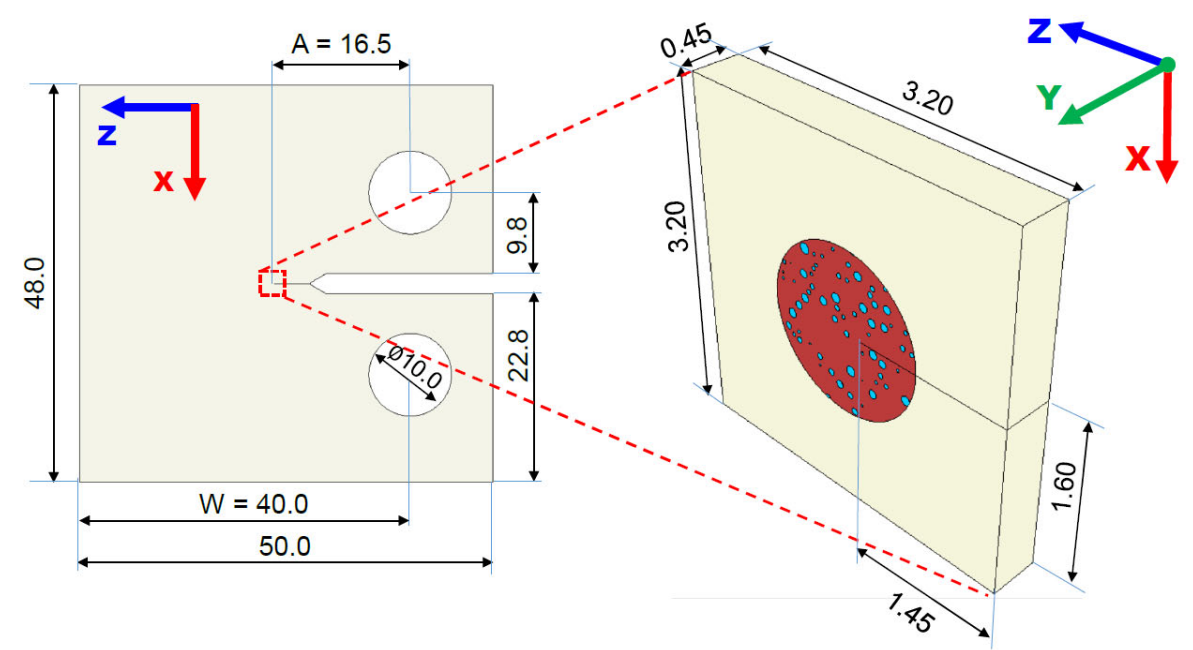

\section{D micro-scale resolved model}

Inner region close-up

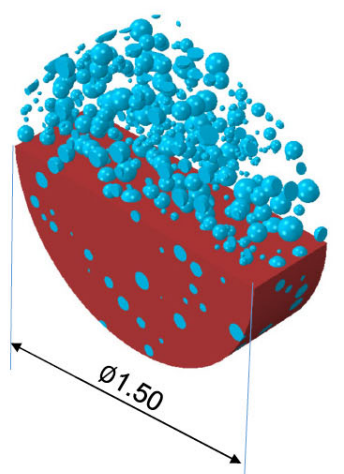

Figure 3 - Multiscale modelling approach used to investigate the cyclic plastic zone in a compact tension specimen made of the composite material described in Section 2. Dimensions are in $\mathrm{mm}$. 


\subsection{Macro-scale model}

The geometry of the compact tension specimen is shown in figure 3. The specimen fulfills the requirements of the ASTM standard E647 [30] and features a pre-crack $4.5 \mathrm{~mm}$ long which extends from the notch in the direction of the Z-axis. Due to symmetry, only the top half of the specimen was meshed in ABAQUS, as shown in figure 4 (a), resulting in $\approx 6000$ quadratic quadrilateral elements. Accordingly, the symmetry condition was imposed at the bottom of the model, i.e. along the symmetry line. The generalized plane strain condition was assumed. The crack was modelled perfectly sharp, as generally recommended in linear kinematic analyses [5], and was inserted in the model by leaving the mesh nodes along the cracked section of the specimen unconstrained. Due to the positive R-values considered in the present work, any mechanical interaction (e.g. contact) between the crack faces was ignored. The loading pin was modelled as a circular rigid surface subjected to a prescribed force per unit thickness $\mathrm{P}$ acting along the X-axis. A surface-to-surface frictionless contact condition was applied between the pin and the specimen's material, enforced via an augmented Lagrange technique.

The constitutive behavior of the macro-scale model mimics the effective response of the composite described in Section 2. Therefore, it was assumed homogeneous and isotropic, with elastic response dictated by either of the two sets of elastic parameters listed in Table 2. To model plastic yielding near the crack tip, the following von Mises-type yield function was used:

$$
f=\sqrt{\frac{3}{2}(\mathbf{s}-\boldsymbol{\alpha}):(\mathbf{s}-\boldsymbol{\alpha})}-R_{p 02}
$$

where $\mathbf{s}$ is the deviatoric part of the Cauchy stress $\boldsymbol{\sigma}, \boldsymbol{\alpha}$ is the back-stress and $R_{p 02}$ is the effective proof stress of the composite. Rate-independent plastic flow was assumed to occur according to the associated flow rule

$$
\dot{\boldsymbol{\varepsilon}}_{\mathbf{p}}=\dot{\lambda} \frac{\partial f}{\partial \boldsymbol{\sigma}}
$$

with $\boldsymbol{\varepsilon}_{\mathbf{P}}, \lambda$ and the operator $\left(^{(}\right)$representing the plastic strain, the plastic multiplier and an infinitesimal increment, respectively. The evolution of the back-stress was described by the relations:

$$
\dot{\boldsymbol{\alpha}}=\sum_{k=1}^{2} \dot{\boldsymbol{\alpha}}_{\mathbf{k}}, \quad \dot{\boldsymbol{\alpha}}_{\mathbf{k}}=\dot{\lambda} H_{k} \frac{\left(\mathbf{s}-\boldsymbol{\alpha}_{\mathbf{k}}\right)}{R_{p 02}}-\dot{\lambda} \gamma_{k} \boldsymbol{\alpha}_{\mathbf{k}}
$$


where the parameters $H_{k}$ and $\gamma_{k}$ describe the kinematic hardening and were determined by fitting the uniaxial response of the RVE, see Figure 2 (b). The fitted values are listed in Table 3. It must be emphasized that the simple model presented above is not meant to describe accurately the effective plastic behavior of the composite material. Its primary aim is only to account for some release of the elastic energy near the crack tip. In this context, an accurate plastic model is not essential. Indeed, the discrepancy between the present plastic model and the real plastic behavior of the material is most likely much smaller than the discrepancy between the present model and a fully elastic solution. In this respect, it was verified that by neglecting the plastic flow completely the variation of the plastic zone size computed inside the microstructure resolved model (see Section 4) was never larger than 4\%.

Constant force amplitude loading of the specimen was simulated by prescribing the time-variation of the force $\mathrm{P}$ on the loading pin. As the plastic zone size never exceeded $0.75 \mathrm{~mm}$ (which is the dimension of the inner region of the micro-scale resolved model, see Section 3.2), the standard relationship between the stress intensity factor $K_{I}$ and the force $\mathrm{P}$ was assumed to apply [30]:

$$
K_{I}=\frac{P}{\sqrt{W}} \frac{(2+\alpha)}{(1-\alpha)^{3 / 2}}\left(0.886+4.64 \alpha-13.32 \alpha^{2}+14.72 \alpha^{3}-5.6 \alpha^{4}\right)
$$

where $\alpha=A / W$, with $A$ and $W$ defined in figure 3. For prescribed values of the maximum stress intensity factor $K_{\text {max }}$ and load ratio $\mathrm{R}$, the model was subjected to five loading cycles in order to obtain a stabilization of the mechanical fields. After that, the displacement data corresponding to the last cycle was extracted and passed on to the micro-scale resolved model, discussed in the next section.

Table 3 - Parameters of the plastic model used to describe the plastic flow of the composite material near the crack tip.

\begin{tabular}{ccccc}
\hline & $H_{1}[\mathrm{GPa}]$ & $\gamma_{1}$ & $H_{2}[\mathrm{GPa}]$ & $\gamma_{2}$ \\
\hline $\mathrm{SGI}$ & 0.21 & 0.01 & 3.16 & 36.3 \\
$\mathrm{Al}-\mathrm{SiC}$ & 2.05 & 0.31 & 2.02 & 0.30 \\
\hline
\end{tabular}




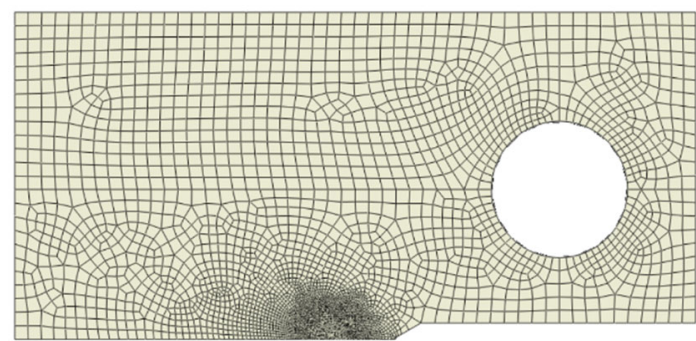

(a)
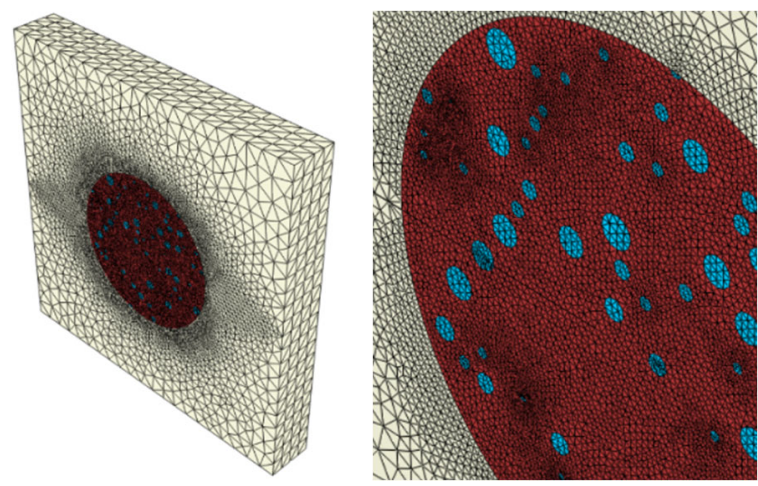

(b)

Figure 4 - Meshes of the finite element models. (a) Macro-scale model. (b) Micro-scale resolved model, with close-up of the inner region.

\subsection{Micro-scale resolved model}

The micro-scale resolved model corresponds to a $3200 \times 450 \times 3200 \mu \mathrm{m}^{3}$ sub-volume of the compact tension specimen (see figure 3), which has its center located $150 \mu \mathrm{m}$ ahead of the crack tip and its faces aligned with the coordinate axes. The model features a cylindrical inner region where the microstructure is fully resolved and a homogenized outer region that represents the effective behavior of the composite. The microstructure in the inner region consists of the metallic matrix with 698 spherical particles, generated based on the diameter and centroid location data presented in Section 2.1. The constitutive behavior of the particles, the matrix and the interface is as described in Section 2.2, whereas the outer region has the same material mechanical behavior of the macro-scale model.

Both the inner and outer regions were meshed in ABAQUS with $\approx 7$ million 4-node hybrid displacement-pressure elements, using a minimum element size of $\approx 10 \mu \mathrm{m}$. The finite element mesh is visualized in figure $4(\mathrm{~b})$. Since the computed size of the plastic zone - which is the main focus of the present work - was always at least ten times larger than the crack opening displacement, the linear kinematic framework was deemed sufficient [5]. The stationary crack was implemented via duplication of the mesh nodes along the crack faces.

It must be emphasized that the thickness of the micro-scale model represents only a fraction of the thickness of the compact tension specimen. Therefore, periodic boundary conditions were applied on the model faces perpendicular to the $\mathrm{Y}$-axis to account for the effect of the surrounding material. That is, for the corresponding points $\boldsymbol{x}^{+}$and $\boldsymbol{x}^{-}$lying on opposite faces, the following conditions were imposed [31]: 


$$
\begin{gathered}
\boldsymbol{u}^{+}=\boldsymbol{u}^{-}+b\left(\varepsilon_{12}^{M} \boldsymbol{e}_{\mathbf{1}}+\varepsilon_{22}^{M} \boldsymbol{e}_{\mathbf{2}}+\varepsilon_{32}^{M} \boldsymbol{e}_{\mathbf{3}}\right) \\
\boldsymbol{t}^{+}=-\boldsymbol{t}^{-}
\end{gathered}
$$

where $\boldsymbol{u}$ is the displacement vector, $\boldsymbol{t}$ is the surface traction, $\boldsymbol{e}_{\boldsymbol{i}}, \mathrm{i}=1,2,3$ are the Cartesian unit vectors along the coordinate axes, $b=450 \mu \mathrm{m}$ is the model thickness and the $\varepsilon_{i j}^{M}, \mathrm{i}, \mathrm{j}=1,2,3$ are the components of the macroscopic strain tensor. The implementation of the boundary conditions (7) on a non-periodic mesh and in the presence of a crack required the development of a special procedure. The procedure, which is based on interpolating the boundary displacement using bicubic Hermite patches, is described in the Appendix.

With regard to the other model faces, i.e. those perpendicular to the X-and Z-axis, the displacement component $u_{y}$ was left unconstrained and the displacement components $u_{x}$ and $u_{z}$ were prescribed based on the displacement data from the 2D macro-scale model. Specifically, $u_{x}$ and $u_{z}$ were set as follows:

$$
\begin{aligned}
& u_{x}(x, z)=\left\{\begin{array}{r}
-u_{x}^{M}\left(\left|x-x_{c t}\right|, z\right) \text { if } x \geq x_{c t} \\
u_{x}^{M}\left(\left|x-x_{c t}\right|, z\right) \text { if } x<x_{c t}
\end{array}\right. \\
& u_{z}(x, z)=u_{z}^{M}\left(\left|x-x_{c t}\right|, z\right)
\end{aligned}
$$

where $x_{c t}$ is the crack tip coordinate along the $\mathrm{X}$-axis and $u_{x}^{M}$ and $u_{z}^{M}$ denote the components of the displacement computed in the macro-scale model. It is noted that the above expression, whose implementation is also discussed in the Appendix, imposes a restriction on the components of the macroscopic strain tensor. Indeed, for two corresponding points $\boldsymbol{x}^{+}$and $\boldsymbol{x}^{-}$located at the edges of the Y-orthogonal faces, both Eq. (7) and Eq. (8) apply. Then, since Eq. (8) states that the two points must have the same values of $u_{x}$ and $u_{z}$, consistency requires that $\varepsilon_{12}^{M}=\varepsilon_{32}^{M}=0$ in Eq. (7). No restriction is placed on $\varepsilon_{22}^{M}$, whose value was left unconstrained in the simulations in order to mimic the situation of generalized plane strain assumed in the macro-scale model.

Upon loading the model, a satisfactory stabilization of the micro-mechanical fields was achieved already with the $2^{\text {nd }}$ cycle, due to the simple constitutive assumptions used for the matrix and the particles and the elastic stress state in the outer region. The plastic zone developing in the inner region of the model was identified based on the normalized cyclic plastic work per unit volume $w_{c, p}$. This quantity was computed as:

$$
w_{c, p}=\frac{1}{w_{p 02}} \int \boldsymbol{\sigma}: \dot{\varepsilon}_{\mathbf{p}}
$$


where the integral was evaluated over the $2^{\text {nd }}$ loading cycle. A point in the matrix was considered "plastic" if $w_{c, p}$ was greater than a threshold $\eta$ set to $10^{-5}$. The definition of the plastic state based on $w_{c, p}$ rather than on the fulfillment of the von Mises criterion has the advantage of revealing the location and magnitude of the so-called active plastic areas [7]. Indeed, attaining the limit of elasticity neither means entry into the plastic regime nor can it indicate the plastic flow intensity.

\section{Results}

\subsection{Shape of the plastic zone}

Figure 5 (a) and (b) show contours of $w_{c, p}$ within two X-Z sections $100 \mu \mathrm{m}$ apart. The data refer to cyclic loading at $K_{\text {max }}=22 \mathrm{MPa} \cdot \mathrm{m}^{0.5}$ and $\mathrm{R}=0.1$, using the material parameters of SGI. To provide a direct comparison with the size of the microstructure, the figure axes, whose origin is at the crack tip, are normalized by the mean distance $a$ between centers of neighboring particles [32]:

$$
a=1 / \sqrt[3]{N}
$$

where $N$ indicates the mean number of particles per unit volume. The contours reveal that the local particle distribution affects to a large extent the plastic zone around the crack tip. In each cross-section, plasticity spreads from the crack tip by linking neighboring particles. This preferential propagation of plasticity along chains of neighboring particles corresponds to experimental observations for different types of monotonic loading $[33,34]$. The impact of the particle spatial arrangement on the local features of the plastic zone is less pronounced when the properties of Al-SiC are considered, altough it remains non-negligible, see figure 5 (c) and (d).

To obtain a more compact $2 \mathrm{D}$ representation of the plasticity distribution in the microstructure, the mean value of $w_{c, p}$ through the model thickness is computed as:

$$
\bar{w}_{c, p}(x, z)=\frac{1}{b-2 \delta_{b}} \int_{\delta_{b}}^{b-\delta_{b}} w_{c, p}(x, y, z) d y
$$

where the parameter $\delta_{b}=30 \mu \mathrm{m}$ is introduced to avoid possible spurious effects near the model boundaries. As before, a point on the X-Z plane is considered plastic if the value of $\bar{w}_{c, p}$ is greater than $\eta$. Figure 6 shows the contour of $\bar{w}_{c, p}$ for both sets of the material parameters. It can be seen that the shape of the plastic region is very irregular. 
Nevertheless, as a whole, the plastic pattern appears symmetric with respect to the crack plane (parallel to the Z-axis), in agreement with the symmetry of the loading and the specimen geometry. This suggests that, with respect to the averaging process of Eq. (11), the thickness of the micro-scale model is sufficient to even out the local variations in the plastic morphology evidenced in Figure 5.

The averaging process described by Eq. (11) facilitates the comparison with predictions based on homogenized material models. In this regard, the model calculations were repeated after replacing the microstructure in the inner region with the corresponding homogenized material properties (the same used in the outer region). The boundary of the contour of $\bar{w}_{c, p}$ computed based on these new calculations is shown with a solid magenta line in figure 6 (a) and (b) and reveals that the homogenized material model noticeably underpredicts the extent of plasticity near the crack. This might relate to the abovementioned tendency of plasticity to propagate preferentially along chains of neighboring particles. Especially in front of the crack, the size of the plastic zone in the microstructure resolved model is substantially larger. This is particularly evident when the parameters of SGI are considered. 


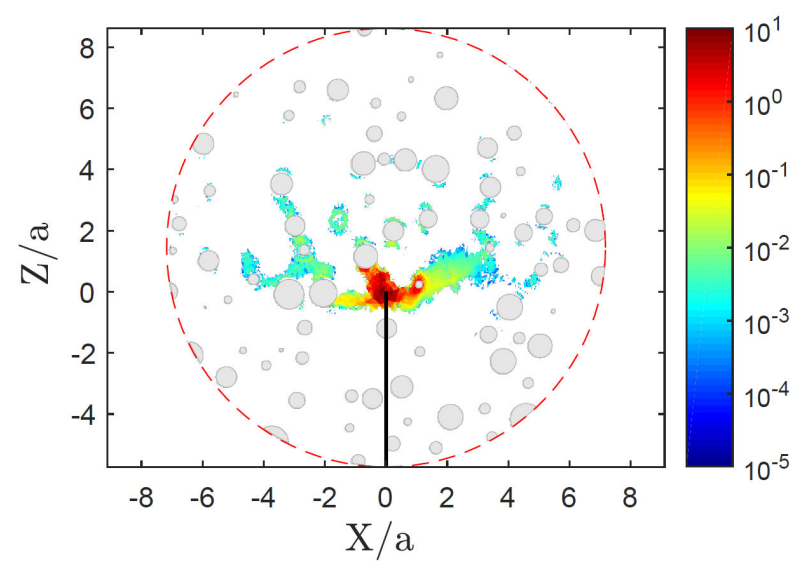

(a)

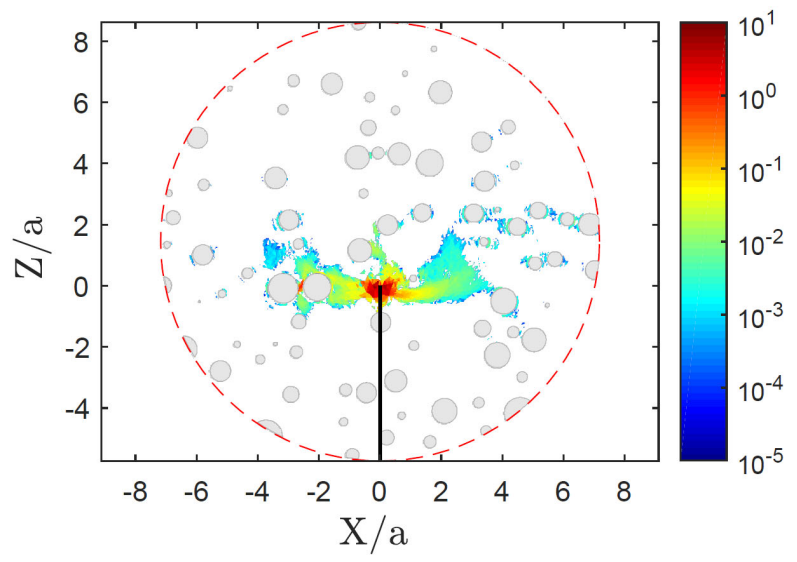

(c)

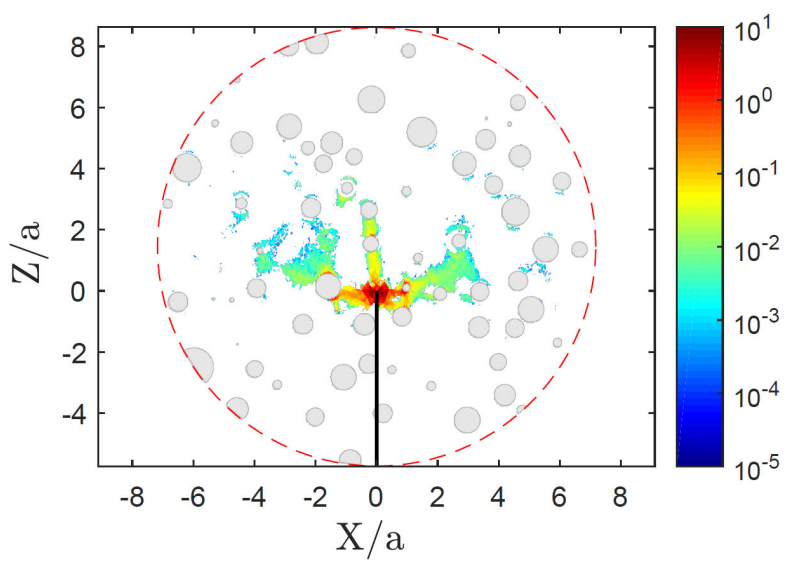

(b)

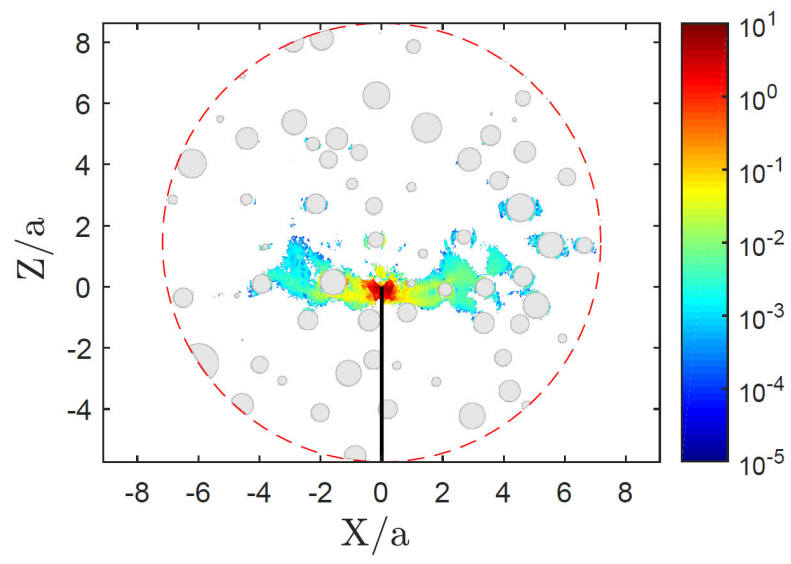

(d)

Figure 5 - Contours of the normalized cyclic plastic work per unit volume $w_{c, p}$ (Eq. (9)) within two X-Z sections of the micro-scale model. The dashed red circle and the solid black line denote the boundary of the inner region and the crack, respectively. (a), (b) Using the parameters of SGI under cyclic loading at $K_{\max }=22 \mathrm{MPa} \cdot \mathrm{m}^{0.5}$ and $\mathrm{R}=0.1$. (c), (d) Using the parameters of Al-SiC under cyclic loading at $K_{\max }=11 \mathrm{MPa} \cdot \mathrm{m}^{0.5}$ and $\mathrm{R}=0.1$. Note that the values of $w_{c, p}$ closest to the crack tip are not reliable, due to the linear kinematic framework. 


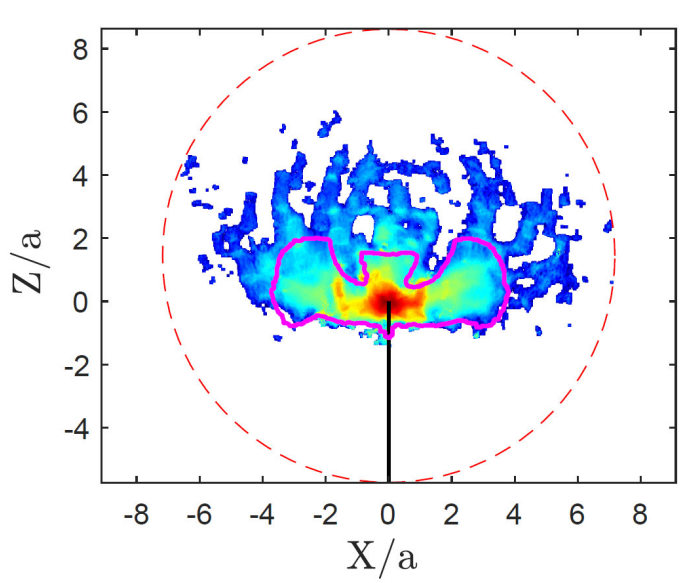

(a)

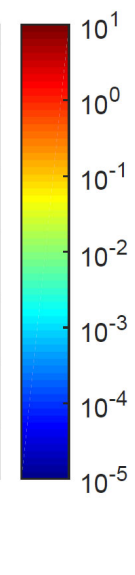

(a)

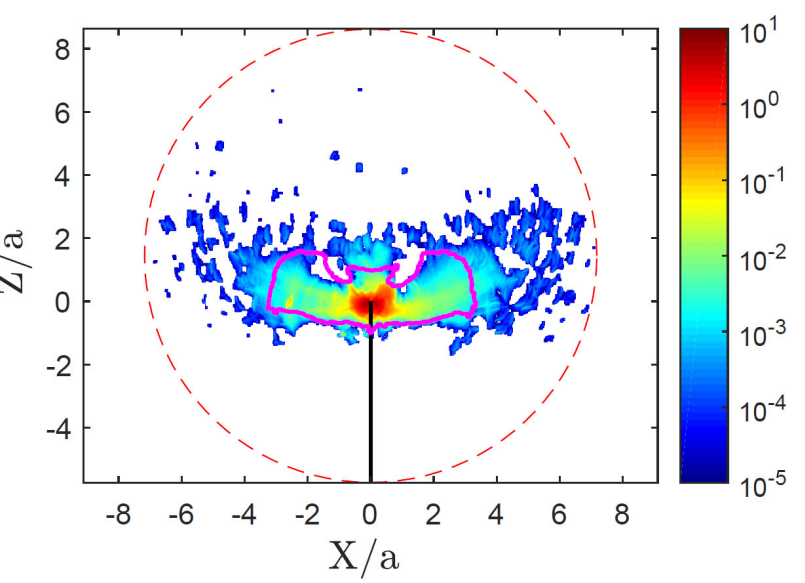

(b)

Figure 6 - Contour of the normalized cyclic plastic work averaged through the thickness of the micro-scale resolved model $\bar{w}_{c, p}$ (Eq. (11)). The dashed red circle and the solid black line denote the boundary of the inner region and the crack, respectively. The solid magenta line represents the boundary of the cyclic plastic zone computed by replacing the inner region with the corresponding homogenized material parameters. (a) SGI, $K_{\max }=22 \mathrm{MPa} \cdot \mathrm{m}^{0.5}, \mathrm{R}=0.1$. (b) Al-SiC, $K_{\max }=11 \mathrm{MPa} \cdot \mathrm{m}^{0.5}, \mathrm{R}=0.1$.

\subsection{Size of the plastic zone}

The contours of $\bar{w}_{c, p}$ reported in figure 6 can be used to define the size of the plastic zone for the composite material under investigation. As mentioned in the introduction, it is common to express the plastic zone size in terms of the distance along the crack growth direction from the crack tip to the plastic boundary. However, the very irregular shape of the plastic zones, as shown in Figure 6, makes this definition ambiguous in the present case. Therefore, it is proposed to use the square root of the plastic zone area as a measure of the plastic zone size. This definition is more robust with respect to the type of data shown in figure 6 and appears physically meaningful due to the relatively small aspect ratio of the plastic zone. On the other hand, it requires modifying Eq. (2) to enable a consistent comparison with the present definition. The simplest way to estimate the area $A_{p}$ of the plastic zone associated with Eq. (2) is by assuming the latter to be a circle with diameter $r_{p, f}$. This assumption is relatively common in the literature, see e.g. [35], and leads to the formula 


$$
\sqrt{A_{p}} \approx \frac{\sqrt{\pi}}{20}\left(\frac{K_{\max }}{R_{p 02}}\right)^{2}
$$

Alternatively, it can be assumed that the shape of the plastic zone is irregular, but such that its area is given by $r_{p, f}^{2}$. This provides

$$
\sqrt{A_{p}} \approx \frac{1}{10}\left(\frac{K_{\max }}{R_{p 02}}\right)^{2}
$$

which is slightly larger than the estimate (12). At this point, it is worth remarking that a well-known expression for the plastic zone shape in a homogeneous material under plane strain is available for monotonic loading [5]. However, such expression is not applicable in the present case, as the plastic zone shape under cyclic loading is different from that under monotonic loading. This is discussed in e.g. Toribio and Kharin [7] and can be appreciated by comparing the plastic zone boundaries shown in Figure 6 with those reported in Anderson [5] for the monotonic case.

The comparison between the plastic zone size predicted via the multiscale model of the compact tension specimen with the SGI material parameters and provided by the analytical estimates presented above is given in figure 7 . The data is normalized by the mean distance $a$ and is plotted over a realistic range of $K_{\max }$ values. The first aspect to note is that the model prediction scales linearly with $K_{\max }^{2}$, in agreement with both Eqs. (12) and (13) and with previous computational studies on homogeneous materials [7]. This could not be taken for granted, given the presence of the particles and therefore of an intrinsic length scale in the heterogeneous material considered. A possible justification is that the particles are distributed quite regularly in the microstructure (see Section 2.1) and therefore the thickness of the model is sufficient to make the effect of the averaging formula (11) equivalent to that of a homogenization procedure.

Figure 7 also shows that, despite the same type of linear dependence on $K_{\max }^{2}$, the plastic zone size computed with the model is almost three times larger than that estimated with either Eq. (12) or Eq. (13). In this respect, it is worth noting that the generalized plane strain assumption tends to increase the plastic zone size compared to the pure plane strain case by allowing a uniform contraction of compact tension specimen in the thickness direction that decreases the level of plastic constraint [36]. However, the specimen remains elastic for the most part during loading and the uniform contraction in the thickness direction is therefore limited. Accordingly, the model prediction does not change appreciably by repeating the calculations under the assumption of pure plane strain - which is at the basis of Eqs. (12) 
and (13). On the other hand, a parameter that affects significantly the results of figure 7 is the threshold $\eta$ (see the definition in Section 3.2). Figure 8 indicates that by increasing $\eta$ the plastic zone sizes computed with the model and estimated with the Eqs. (12) and (13) can be made to coincide. However, this happens when $\eta$ is about $1 \times 10^{-2}$. This value is physically unrealistic, as it would imply that a point in the elastic domain just outside the plastic zone would experience, after as few as 100 cycles, a plastic work equivalent to $w_{p 02}$. Therefore, although the choice of $\eta$ does play a role, it is not sufficient to justify the discrepancy. In the light of these considerations, it can be concluded that the analytical expressions (12) and (13), which are based on Eq. (2), are not optimal to estimate the plastic zone size in a material like the composite considered here.

It is also worth examining the comparison with the plastic zone size predicted by the compact tension specimen model upon replacement of the microstructure with the homogenized material. Figure 7 reveals that such a fully homogenized model underestimates the plastic zone size by approx. $40 \%$ compared to the microstructure resolved model, thus confirming the impression gained from figure 6. The poor performance of the homogenized model may stem from several factors. One is its inability to capture the shape of the plastic zone in front of the crack - see the previous section and Figure 6 - which supports the claims mentioned in the introduction that the associated von Mises plasticity is not the best choice for metals containing particles [8,9]. Another factor is the continuous yielding behavior of the composite, well-known for similar materials $[11,20]$, which is in contrast with the common modelling assumption of a sharp threshold for plastic flow.

Compared to the microstructure resolved model, the prediction of the homogenized model lies relatively closer to the analytical estimates (12) and (13). This is not surprising, as both assume material homogeneity and plastic flow driven by the von Mises stress. In this context, the discrepancy between the homogenized model and the former analytical estimates is likely to arise from the assumptions on the plastic zone shape introduced to derive Eqs. (12) and (13). Strain hardening of the homogenized material and the threshold $\eta$ can also play a role by affecting the prediction of the homogenized model. However, the use of a finite threshold for plasticity detection is expected to lead to an underestimation of the plastic zone size. Similarly, strain hardening should limit the extent of the plastic flow, as the material can carry higher stresses close to the crack tip. On the contrary, Figure 7 shows that the estimate of the plastic zone size based on the homogenized model is actually larger than the analytical prediction. Therefore, the main cause of the discrepancy can be neither the strain hardening nor the finite threshold $\eta$, but rather the assumption on the plastic zone shape. 


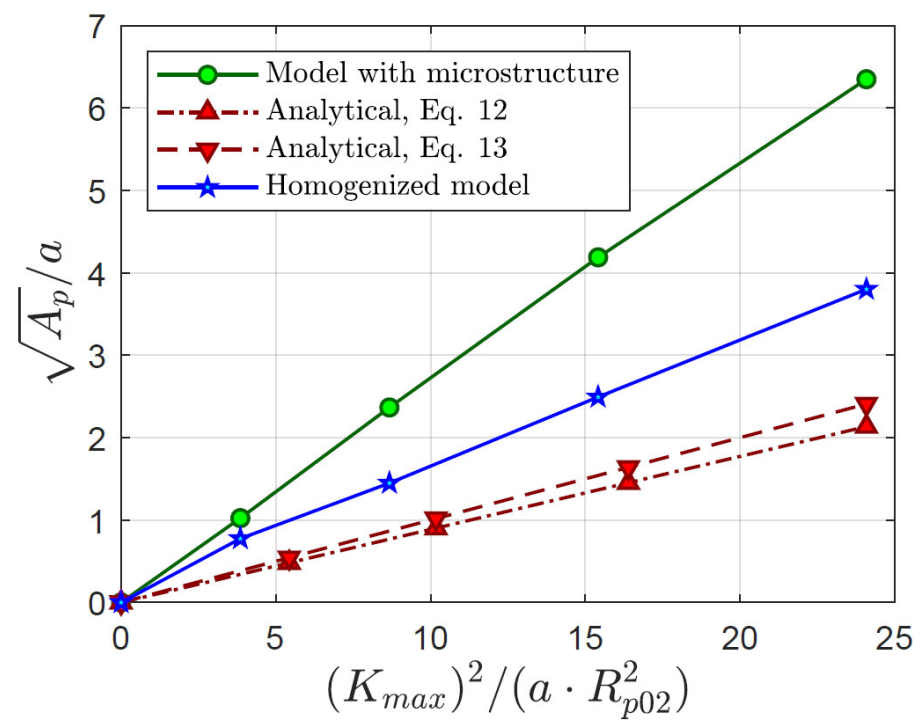

Figure 7 - Plastic zone size as a function of the maximum stress intensity factor during cylic loading. The values based on the multiscale model of the compact tension specimen with resolved microstructure are compared to the analytical estimates (12) and (13) as well as to the prediction of the model with the microstructure replaced by homogenized material. The data refer to the properties of SGI and $\mathrm{R}=0.1$.

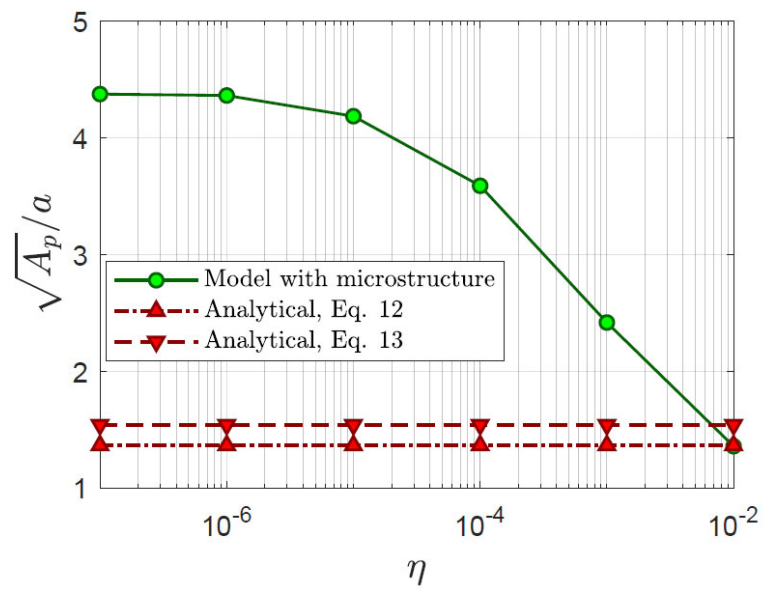

Figure 8 - Effect of the threshold $\eta$ on the plastic zone size computed with the multiscale model with resolved microstructure. The material properties of SGI are used, combined with loading at $\mathrm{R}=0.1$ and $K_{\max }=18 \mathrm{MPa} \cdot \mathrm{m}^{0.5}$. 


\subsection{Sensitivity to the model parameters}

The framework of dimensional analysis [37] is employed to analyze the results discussed in the previous section and in particular the dependence of the computed plastic zone size on the choice of the model parameters. Accordingly, the plastic zone size is written as a generic function of the model parameters:

$$
\sqrt{A_{p}}=F\left(\sigma_{y}^{m}, E^{m}, v^{m}, E^{p}, v^{p}, R, K_{\max }, a, f_{V}, \Xi\right)
$$

In this expression, the first five quantities are associated with the constitutive behavior of the matrix and the particles, $R$ and $K_{\max }$ relate to the loading and the remaining quantities describe the topology of the microstructure. In particular, $\Xi$ characterizes the particle size and spatial distribution and can be thought of as a set of coefficients that provide the location and diameter of the particles near the crack.

In Eq. (14), the dependence on $\sigma_{y}^{m}$ is inconvenient, as this quantity can vary significantly within the same material family and is often difficult to measure. Therefore, it is advantageous to replace $\sigma_{y}^{m}$ with $R_{p 02}$, the proof stress of the entire composite, which can be easily measured experimentally (or obtained numerically by virtual testing on an RVE). This can be done because, for the material investigated here, a monotonic relationship exists between these two quantities. Consequently, Eq. (14) can be re-written as:

$$
\sqrt{A_{p}}=F^{\prime}\left(R_{p 02}, E^{m}, v^{m}, E^{p}, v^{p}, R, K_{\max }, a, f_{V}\right)
$$

where the dependence on $\Xi$ has been dropped since the possibility of modifying the particle size and spatial distributions was not explored in the present work.

The expression (15) involves ten quantities and two independent dimensions: length and stress. Therefore, according to the Buckingham's pi-theorem, it can be re-formulated in terms of eight dimensionless groups, which must be chosen such that they form a basis of the null space of the corresponding dimension matrix [37]. Here, the following choice is made:

$$
\frac{\sqrt{A_{p}}}{a}=\Psi\left(\frac{K_{\max }^{2}}{a R_{p 02}^{2}}, R, \frac{E^{m}}{R_{p 02}}, v^{m}, \frac{E^{p}}{E^{m}}, v^{p}, f_{V}\right)
$$

This equation shows that the sensitivity to the model parameters can be investigated simply by assessing the dependence of the data in figure 7 on the variation of the other dimensionless groups appearing as arguments of $\Psi$. In 
this regard, figure 9 demonstrates that neither an increase of $\mathrm{R}$, nor variations of the ratio $E^{m} / R_{p 02}$ within a realistic range have significant impact on the computed size of the plastic zone. That is, the function $\Psi$ is essentially insensitive to the values of these two dimensionless groups. On the other hand, figure 10 (a) reveals that the particle volume fraction - which was varied in the model by modifying the diameter of all the particles by the same relative amount has a strong effect on the extent of plasticity. In particular, for the same effective proof stress and loading conditions, the larger the particle volume fraction, the larger the size of the plastic zone. This fact is independent of the choice of the elastic and plastic parameters, as figure 10 (b) demonstrates that the same effect is seen when the properties of Al$\mathrm{SiC}$ are considered. It can also be observed that, for the considered range of particle volume fractions, the discrepancy with the analytical estimates (12) and (13) becomes larger, resp. smaller, as the particle volume fraction increases, resp. decreases. In particular, the discrepancy is minimal when the matrix material alone is considered $\left(f_{V}=0 \%\right)$, which is to be expected as this situation corresponds to the hypotheses of homogeneity and von Mises based plastic flow used to obtain Eqs. (12) and (13).

The comparison between figure 10 (a) and figure 10 (b) also reveals that the plastic zone size can vary by up $\approx 15 \%$ when the values of the dimensionless groups that control the elastic response of the particles and the matrix are changed from those corresponding to SGI to those corresponding to Al-SiC. Considering the large difference between the parameters of these two materials $\left(E^{p} / E^{m} \approx 7.3 \times 10^{-2}\right.$ for SGI and $E^{p} / E^{m} \approx 5.5$ for Al-SiC, see Table 1$)$ it is remarkable that its effect on the values of $\Psi$ is rather small.

In addition to the parameters just discussed, the influence of minor deviations of the particle geometry from the spherical shape was evaluated. To this purpose, a model similar to the one described in Section 0 was developed where each particle was modelled as an ellipsoid of revolution. The length and orientation of the ellipsoid's axes were set based on the requirements of achieving normalized second central moments equal to those of the graphite nodule obtained from the X-ray tomography data (see Section 2.1). A Matlab function was used to this purpose [38], which produced ellipsoids having a mean value of the ratio between the lengths of the maximum and minimum axes equal to 1.34 . To facilitate the meshing of the model, a volume fraction of $6 \%$ instead of $11 \%$ was used. Figure 11 compares the plastic zone sizes predicted by the multiscale microstructure resolved model with either spherical or ellipsoidal particles. Only minor differences can be detected, indicating that the previous findings are robust with respect to minor deviations from the spherical shape. 


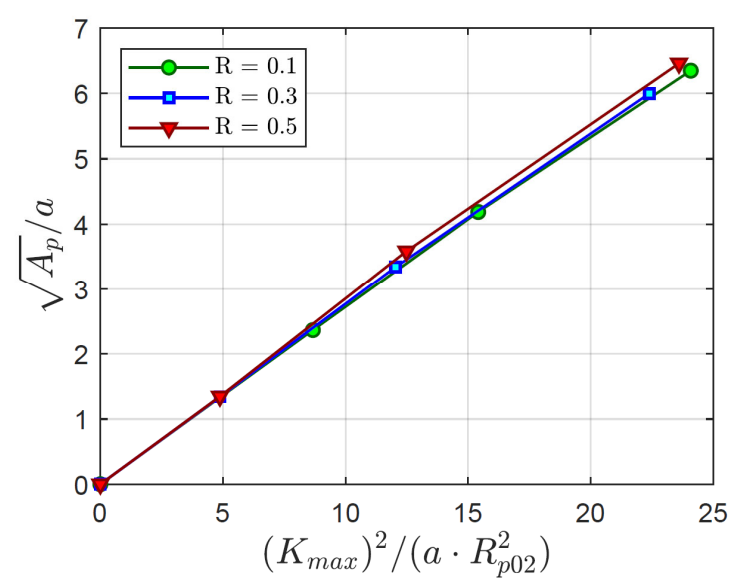

(a)

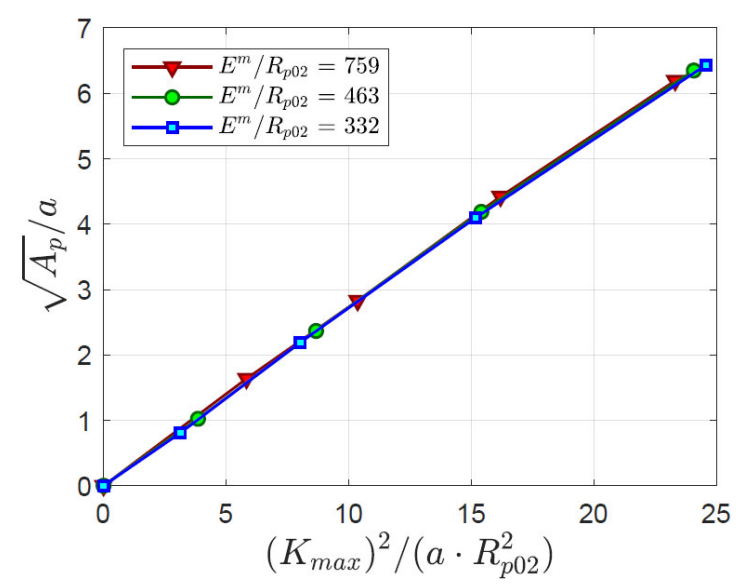

(b)

Figure 9 - Effect of the model parameters on the relationship between the plastic zone size and maximum stress intensity factor obtained using the microstructure resolved multiscale model. (a) Effect of the load ratio R. (b) Effect of the ratio between the matrix Young's modulus $E^{m}$ and the composite proof stress $R_{p 02}$.

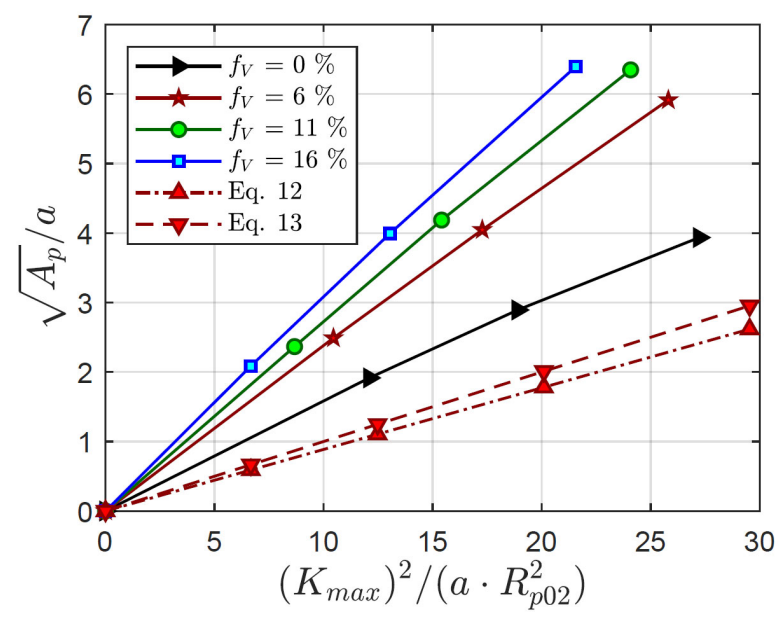

(a)

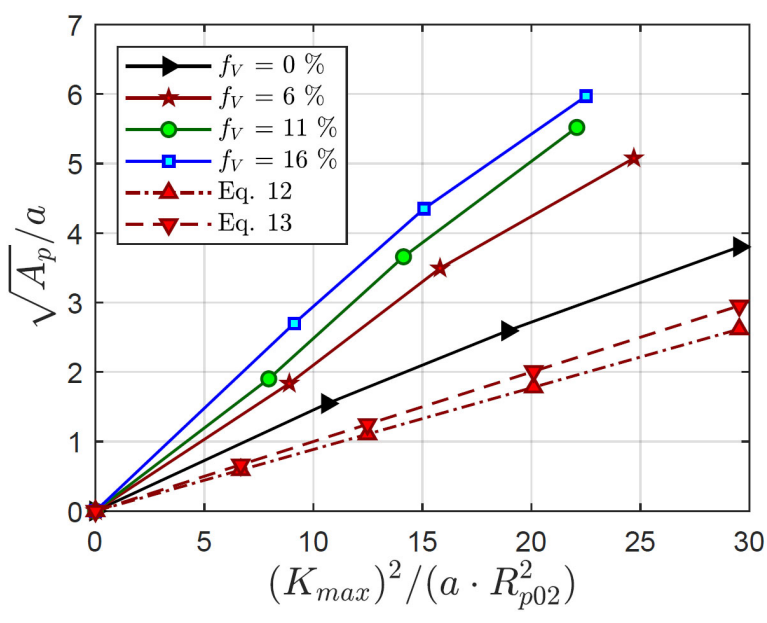

(b)

Figure 10 - Effect of the particle volume fraction on the relationship between the plastic zone size and maximum stress intensity factor obtained using the microstructure resolved multiscale model. (a) Material properties of SGI. (b) Material properties of $\mathrm{Al}-\mathrm{SiC} . \mathrm{R}=0.1$ is considered. 


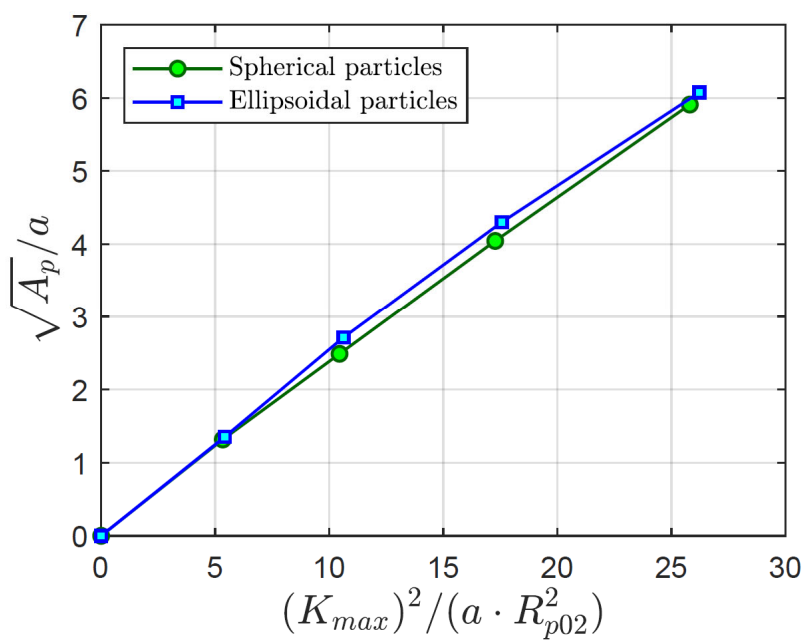

Figure 11 - Comparison between the plastic zone sizes computed assuming either spherical or ellipsoidal shape of the particles in the microstructure resolved multiscale model. The data refer to the properties of SGI, R= 0.1 and $f_{V}=6 \%$.

\section{Simplified formula to estimate the plastic zone size}

The previous section has shown that the plastic zone size computed with the multiscale model can be substantially larger compared to the analytical estimates (12) and (13). To compensate for this, an improved formula is proposed here that accounts for the effect of the particle volume fraction while retaining the simple structure of the former expressions.

The starting point is to note is that the plots in Figure 9 - Figure 11 indicate that the relationship between the plastic zone size normalized by the mean particle distance $\sqrt{A_{p}} / a$ and $K_{\max }^{2} / a R_{p 02}^{2}$ remains approximately linear for all investigated choices of the model parameters. This means that the dependence on $a$ can be neglected in Eq. (15) and, consequently, Eq. (16) can be rewritten as

$$
\sqrt{A_{p}} \approx \psi\left(R, \frac{E^{m}}{R_{p 02}}, v^{m}, \frac{E^{p}}{E^{m}}, v^{p}, f_{V}\right)\left(\frac{K_{\max }}{R_{p 02}}\right)^{2}
$$

Furthermore, the sensitivity analysis of Section 4.3 shows that the dependence on $R$ and $E^{m} / R_{p 02}$ is negligible. Also, the effect of $E^{p} / E^{m}, v^{m}$ and $v^{p}$ is minor in comparison to that of $f_{V}$. Based on these considerations, Eq. (17) can be simplified to 


$$
\sqrt{A_{p}} \approx \psi\left(f_{V}\right)\left(\frac{K_{\max }}{R_{p 02}}\right)^{2}
$$

Finally, an expression for $\psi\left(f_{V}\right)$ can be obtained by computing the mean slope of the curve $\sqrt{A_{p}} / a$ vs. $K_{\text {max }}^{2} /\left(a R_{p 02}^{2}\right)$ in the considered interval $[0,25]$ (see Figure 10) for several values of $f_{V}$. The outcome is plotted in figure 12, where the interval bars correspond to the difference in material properties of SGI (upper limit) and Al-SiC (lower limit). Based on the data in the figure, the following second order polynomial is used to approximate the function $\psi\left(f_{V}\right)$ :

$$
\psi\left(f_{V}\right) \approx-2.63 f_{V}^{2}+1.30 f_{V}+0.146
$$

Expression (18), combined with Eq. (19), provides an estimate of the size of the crack tip plastic zone during mode I constant amplitude loading within the range of the considered parameters of the idealized composite material described in Section 2.

As anticipated, the structure of Eq. (18) is similar to that of the well-known estimate (2), which is based on Irwin's analysis. However, two important differences exist. First, the size of the plastic zone in Eq. (18) is expressed in terms of the square root of the plastic area instead of the distance between the crack tip and the plastic boundary in front of it. Second, the proportionality factor between the plastic zone size and $K_{\max }^{2} / R_{p 02}^{2}$ is not a constant but depends on the particle volume fraction. This point is crucial, as failure to account for the effect of the volume fraction may result in a significant underestimation of the plastic zone size, as discussed in Section 4.

The appeal of Eq. (18) is enhanced by the poor performance of the model with microstructure replaced by homogenized material discussed previously. Such a model, which rests on the simplifying hypotheses of homogeneity, von Mises-based associated plastic flow and sharp yield threshold, is unable to account for the strong effect played by the particle volume fraction. More advanced plasticity models would probably be able to capture the effect better, but they would inevitably require an additional time investment.

At this point, a few remarks regarding the applicability of Eq. (18) to real materials are needed. Based on the composite material definition in Section 2, the formula (18) is applicable to metals containing spherical elastic particles, although minor deviations from the spherical shape are allowed. Metal hardening, limited interface strength and presence of residual stresses were all neglected in the present analysis, even though the normalization by $R_{p 02}$ at least partially accounts for these effects and thus the applicability range of the present findings is possibly broader than stated here. In addition, the particle size and spatial distribution considered in this work were representative of SGI. The analysis 
to a metal matrix composite was ad-hoc extended by merely replacing the material properties. However, the microstructure of cast iron has already been used in the literature as a model for metal matrix composites [39], thus suggesting the applicability of Eq. (18) to at least these two material classes. Concerning the particle volume fraction, the non-linearity of the function $\psi\left(f_{V}\right)$ discourages the direct extrapolation of Eq. (18) for values of $f_{V}$ outside the interval considered in figure 12. Negative values of $\mathrm{R}$ must be avoided as well, as the interaction between the crack faces was neglected in the model. Finally, the size of the particles must be relatively large (in any case larger than 1 micron) such that the matrix-particle interface energy remains negligible, dislocation-related size effects are not triggered and the hypothesis of isotropic behavior of the matrix is fulfilled. Future experimental studies based on, for example, direct measurements of the dislocation density near the crack tip [40] could help further elucidate the accuracy and applicability of the present results.

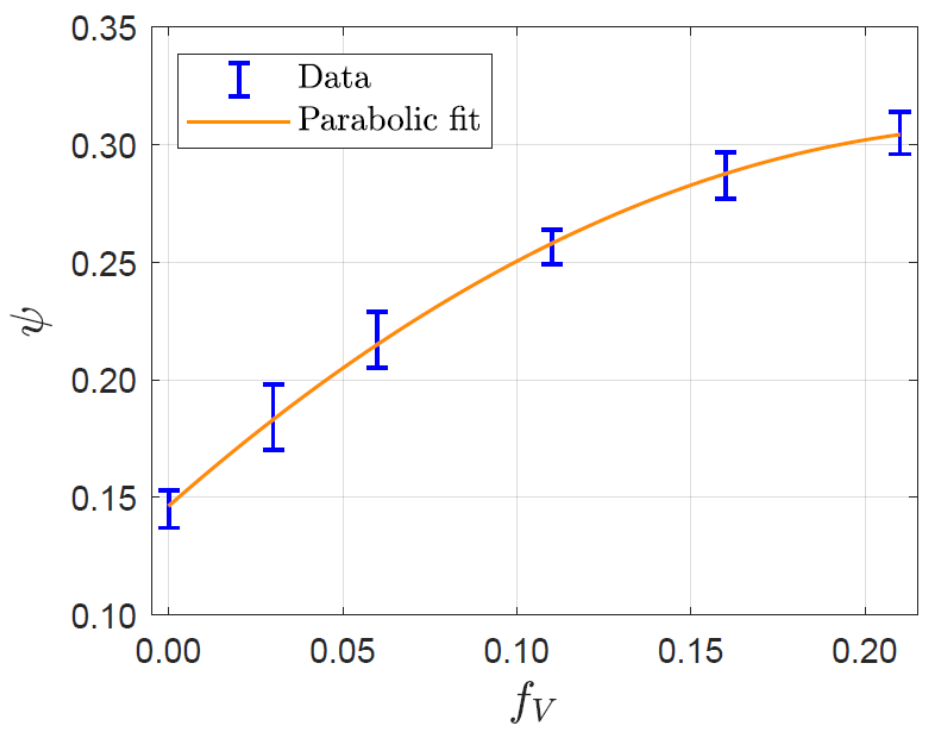

Figure 12 - Mean slope of the $\sqrt{A_{P}} / a$ vs. $K_{\max }^{2} /\left(a R_{p 02}^{2}\right)$ curves computed with the microstructure resolved multiscale model as a function of the particle volume fraction. The interval bars correspond to the variation due to considering either the material properties of SGI (upper limit) or those of Al-SiC (lower limit). 


\section{Conclusions}

The multiscale model with resolved microstructure shows that the presence of the particles modifies the shape and increases the size of the cyclic plastic zone compared to what is expected based on the hypotheses of material homogeneity and associated von Mises plasticity. The scaling of the plastic zone size with the square of the maximum stress intensity factor is confirmed, but the proportionality coefficient is strongly affected by the particle volume fraction. In particular, for the same effective proof stress and loading conditions, a higher particle volume fraction results in a larger cyclic plastic zone. The well-known Irwin's formula cannot account for this effect and therefore, it may underestimate the extent of plasticity by as much as a factor of 3 for a particle volume fraction of $\approx 20 \%$. To compensate for this, a new compact formula is proposed that provides an estimate of the size of the cyclic plastic zone in metals containing small to medium volume fractions of elastic particles. The formula, which is based on a number of numerical simulations, holds for material properties ranging from metal matrix composites to cast irons and is robust to minor particle deviations from the idealized spherical shape.

\section{Acknowledgements}

This work received support from the Danish Research Council for Independent Research, grant no. 8022-00085B. T.A. gratefully acknowledges Prof. Jesper Hattel for funding acquisition and Senior Researcher Søren Fæster for sharing the X-ray tomography data.

\section{Appendix: Implementation of the boundary conditions for the micro-}

\section{scale resolved model}

There are two challenges associated with the implementation of the boundary conditions specified by Eqs. (7) and (8) in the micro-scale resolved model. The first is that the finite element mesh is not symmetric. This means that it is not possible to establish a one-to-one correspondence between the nodes lying on opposite faces of the model, which would greatly simplify the imposition of the periodic conditions (7). Some methods exist to overcome this issue, which are typically based on interpolating the boundary displacement via proper interpolants and then using the latter to enforce periodicity. For instance, Nguyen et al. [26] proposed to interpolate the displacement on a given face via a bilinearly blended Coons patch of polynomials defined along the face edges. However, this technique is not applicable 
in the presence of discontinuities such as cracks, which is the second challenge in the present case. The method of Akpoyomare et al. [27], which is based on decomposing a face into triangles over which linear interpolants are defined, does not suffer from this limitation. On the other hand, to decrease the impact of the non-smooth transition at the edges of the triangles, a high number of triangles should be used, thus increasing the computational complexity. To obtain an interpolation that is smooth and allows for the presence of discontinuities, bicubic Hermite patches were used in the present work. This approach is explained in the following.

First, the model face whose outward normal points in the negative direction of the Y-axis, denoted by $\partial \Omega^{Y-}$, is subdivided into a $n_{x} \times n_{z}$ collection of rectangular patches. While doing so, care is taken to make sure that the crack runs in between the patches, without crossing any of them. The boundary displacement inside each rectangular domain $\left[x_{i-1}, x_{i}\right] \times\left[z_{j-1}, z_{j}\right] \subset \partial \Omega^{Y-}$ that corresponds to the patch $S_{i, j}$ is then interpolated as:

$$
\widetilde{\boldsymbol{u}}(x, z)=\sum_{k, m=1}^{4} B_{k m}^{i, j} h_{k}(x) h_{m}(z)
$$

The functions $h_{k}(s)$ appearing in the last expression are standard cubic Hermite interpolants, which are defined over the generic interval $\left[s_{i-1}, s_{i}\right]$ as:

$$
\begin{gathered}
h_{1}(s)=1-3 \xi^{2}+2 \xi^{3} \\
h_{2}(s)=l\left(\xi-2 \xi^{2}+\xi^{3}\right) \\
h_{3}(s)=3 \xi^{2}-2 \xi^{3} \\
h_{4}(s)=l\left(-\xi^{2}+\xi^{3}\right)
\end{gathered}
$$

with $l=s_{i}-s_{i-1}$ and $\xi=\left(s-s_{i-1}\right) / l$. Concerning the terms $B_{k m}^{i, j}$, they correspond to the values of the displacement and displacement derivatives at the corners of the patch and can be represented by the following $4 \times 4$ matrix

$$
\underline{B}^{i, j}=\left[\begin{array}{cccc}
u^{i-1, j-1} & \boldsymbol{u}_{, z}^{i-1, j-1} & \boldsymbol{u}^{i-1, j} & \boldsymbol{u}_{, z}^{i-1, j} \\
\boldsymbol{u}_{, x}^{i-1, j-1} & \boldsymbol{u}_{, x z}^{i-1, j-1} & \boldsymbol{u}_{, x}^{i-1, j} & \boldsymbol{u}_{, x z}^{i-1, j} \\
\boldsymbol{u}^{i, j-1} & \boldsymbol{u}_{, z}^{i, j-1} & \boldsymbol{u}^{i, j} & \boldsymbol{u}_{, z}^{i, j} \\
\boldsymbol{u}_{, x}^{i, j-1} & \boldsymbol{u}_{, x z}^{i, j-1} & \boldsymbol{u}_{, x}^{i, j} & \boldsymbol{u}_{, x z}^{i, j}
\end{array}\right]
$$

where the following notation has been adopted: 


$$
\begin{array}{ll}
\boldsymbol{u}^{i, j}=\boldsymbol{u}\left(x_{i}, z_{j}\right), & \boldsymbol{u}_{, \boldsymbol{x}}^{i, j}=\frac{\partial \boldsymbol{u}}{\partial x}\left(x_{i}, z_{j}\right) \\
\boldsymbol{u}_{, z}^{i, j}=\frac{\partial \boldsymbol{u}}{\partial z}\left(x_{i}, z_{j}\right), & \boldsymbol{u}_{, x z}^{i, j}=\frac{\partial^{2} \boldsymbol{u}}{\partial x \partial z}\left(x_{i}, z_{j}\right)
\end{array}
$$

Expression (A.1) defines a bicubic Hermite patch. The notable feature of interpolating the boundary displacement in terms of a collection of bicubic Hermite patches is that the interpolated displacement, its first derivatives and the mixed $2^{\text {nd }}$-order derivative are all continuous across adjacent patches. In addition, the discontinuity associated with the crack can be easily handled by "duplicating" the patch vertices along the crack path, as usually done with standard finite element meshes.

With the displacement interpolation $\widetilde{\boldsymbol{u}}(x, z)$ at hand, the boundary condition (7) can be written as:

$$
\boldsymbol{u}=\left\{\begin{array}{lll}
\widetilde{\boldsymbol{u}}(x, z) & \text { if } & \boldsymbol{x} \in \partial \Omega^{Y-} \\
\widetilde{\boldsymbol{u}}(x, z)+b \varepsilon_{22}^{M} \boldsymbol{e}_{2} & \text { if } & \boldsymbol{x} \in \partial \Omega^{Y+}
\end{array}\right.
$$

The implementation of Eq. (A.5) in the micro-scale model involves constraining the displacement of each mesh node on $\partial \Omega^{Y-}$ and on $\partial \Omega^{Y+}$ to the relevant degrees of freedom of $\widetilde{\boldsymbol{u}}(x, z)$ - which are the values of the displacement and its derivatives at the patch vertices, see Eq. (A.3). The procedure is analogous to that discussed in ref. [27] for a piecewise linear interpolant and, for the sake of brevity, is not repeated here.

The boundary condition (8) can also be implemented by means of the $\widetilde{\boldsymbol{u}}(x, z)$ interpolant. Indeed, since the displacement components $u_{x}$ and $u_{z}$ on the model faces perpendicular to the X-and Z-axis are assumed independent of the y-coordinate, they can be expressed in terms of $\widetilde{\boldsymbol{u}}(x, z)$ evaluated along the edges of $\partial \Omega^{Y-}$. Again, this involves constraining the displacement of the mesh nodes to the relevant degrees of freedom of $\widetilde{\boldsymbol{u}}(x, z)$. Next, to enforce Eq. (8), the degrees of freedom of $\widetilde{\boldsymbol{u}}(x, z)$ that control $\tilde{u}_{x}$ and $\tilde{u}_{z}$ along the edges of $\partial \Omega^{Y-}$ are prescribed based on $u_{x}^{M}$ and $u_{z}^{M}$, i.e. the components of the displacement in the macro-scale model.

\section{References}

[1] P. Chowdhury, H. Sehitoglu, Mechanisms of fatigue crack growth - a critical digest of theoretical developments, Fatigue Fract. Eng. Mater. Struct. 39 (2016) 652-674. doi:10.1111/ffe.12392.

[2] U. Zerbst, M. Madia, M. Vormwald, H.T. Beier, Fatigue strength and fracture mechanics - A general perspective, Eng. Fract. Mech. 198 (2018) 2-23. doi:10.1016/j.engfracmech.2017.04.030. 
[3] G.R. Irwin, Plastic zone near a crack and fracture toughness, Sagamore Res. Conf. Proc. 4 (1961) 63-78.

[4] D.S. Dugdale, Yielding in steel sheets containing slits, J. Mech. Phys. Solids. 8 (1960) 100-104.

[5] T.L. Anderson, Fracture mechanics - Fundamentals and applications, Third Edit, CRC Press, Boca Raton, 2005.

[6] J.R. Rice, Mechanics of crack tip deformation and extension by fatigue, ASTM STP 415. (1967) 247.

[7] J. Toribio, V. Kharin, Finite-deformation analysis of the crack-tip fields under cyclic loading, Int. J. Solids Struct. 46 (2009) 1937-1952. doi:10.1016/j.ijsolstr.2009.01.006.

[8] C.J. Lissenden, Experimental yield surface determination for metal matrix composites, Solid Mech. Its Appl. 168 (2010) 173-185. doi:10.1007/978-90-481-3467-0_13.

[9] M. Metzger, T. Seifert, Computational assessment of the microstructure-dependent plasticity of lamellar gray cast iron - Part II : initial yield surfaces and directions, Int. J. Solids Struct. 66 (2015) 194-206. doi:10.1016/j.ijsolstr.2015.04.014.

[10] Z.H.A. Kassam, R.J. Zhang, Z. Wang, Finite element simulation to investigate interaction between crack and particulate reinforcements in metal-matrix composites, Mater. Sci. Eng. A. 203 (1995) 286-299.

[11] A. Miserez, A. Rossoll, A. Mortensen, Investigation of crack-tip plasticity in high volume fraction particulate metal matrix composites, Eng. Fract. Mech. 71 (2004) 2385-2406. doi:10.1016/j.engfracmech.2004.01.006.

[12] D.K. Yi, Z.M. Xiao, S.K. Tan, Y.M. Zhang, Elastic-plastic fracture behavior of a radial matrix crack interacting with a circle inclusion with generalized Irwin corrections, Acta Mech. 225 (2014) 91-107. doi:10.1007/s00707-013-0954-7.

[13] M. Fan, D.K. Yi, Z.M. Xiao, A Zener-Stroh crack in fi ber-reinforced composites with generalized Irwin plastic zone correction, Int. J. Mech. Sci. 82 (2014) 81-89. doi:10.1016/j.ijmecsci.2014.03.008.

[14] B.B. Bouiadjra, M. Elmeguenni, M. Benguediab, M. Belhouari, M. Nait-abdelaziz, Numerical estimation of the effects of microcavities on the plastic zone size ahead of the crack tip in aluminum alloy 2024 T3, Mater. Des. 30 (2009) 752-757. doi:10.1016/j.matdes.2008.05.021.

[15] S.K. Paul, Effect of micro-cavities on different plastic zones at the fatigue crack tip of a compact tension specimen, Eng. Fract. Mech. 158 (2016) 13-22. doi:10.1016/j.engfracmech.2016.02.041.

[16] S.K. Paul, Numerical models to determine the effect of soft and hard inclusions on different plastic zones of a fatigue crack in a C(T) specimen, Eng. Fract. Mech. 159 (2016) 90-97. 
doi:10.1016/j.engfracmech.2016.03.028.

[17] V. Tvergaard, J.W. Hutchinson, The relation between crack growth resistance and fracture process parameters elastic-plastic solids, J. Mech. Phys. Solids. 40 (1992) 1377-1397.

[18] V. Tvergaard, J.W. Hutchinson, Two mechanisms of ductile fracture : void by void growth versus multiple void interaction, Int. J. Solids Struct. 39 (2002) 3581-3597.

[19] G. Hütter, L. Zybell, M. Kuna, Micromechanical modeling of crack propagation in nodular cast iron with competing ductile and cleavage failure, Eng. Fract. Mech. 147 (2015) 388-397. doi:10.1016/j.engfracmech.2015.06.039.

[20] T. Andriollo, Y.B. Zhang, S. Fæster, J. Thorborg, J. Hattel, Impact of micro-scale residual stress on in-situ tensile testing of ductile cast iron: Digital volume correlation vs. model with fully resolved microstructure vs. periodic unit cell, J. Mech. Phys. Solids. 125 (2019) 714-735. doi:10.1016/J.JMPS.2019.01.021.

[21] T. Andriollo, Y. Zhang, S. Fæster, V. Kouznetsova, Analysis of the correlation between micro-mechanical fields and fatigue crack propagation path in nodular cast iron, Acta Mater. 188 (2020) 302-314. doi:https://doi.org/10.1016/j.actamat.2020.02.026.

[22] N.A.C. Cressie, Statistics for spatial data, Revised ed, John Wiley \& Sons, New York, 1993.

[23] M.A. Azeem, M.K. Bjerre, R.C. Atwood, N. Tiedje, P.D. Lee, Synchrotron quantification of graphite nodule evolution during the solidification of cast iron, Acta Mater. 155 (2018) 393-401. doi:10.1016/j.actamat.2018.06.007.

[24] T. Andriollo, S. Fæster, G. Winther, Probing the structure and mechanical properties of the graphite nodules in ductile cast irons via nano-indentation, Mech. Mater. $122 \quad$ (2018) 85-95. doi:10.1016/j.mechmat.2018.03.010.

[25] V. V. Ganesh, N. Chawla, Effect of particle orientation anisotropy on the tensile behavior of metal matrix composites: Experiments and microstructure-based simulation, Mater. Sci. Eng. A. 391 (2005) 342-353. doi:10.1016/j.msea.2004.09.017.

[26] V.-D. Nguyen, E. Béchet, C. Geuzaine, L. Noels, Imposing periodic boundary condition on arbitrary meshes by polynomial interpolation, Comput. Mater. Sci. 55 (2012) 390-406. doi:10.1016/j.commatsci.2011.10.017.

[27] A.I. Akpoyomare, M.I. Okereke, M.S. Bingley, Virtual testing of composites : Imposing periodic boundary conditions on general finite element meshes, Compos. Struct. 160 (2017) 983-994. 
doi:10.1016/j.compstruct.2016.10.114.

[28] T. Sjögren, I.L. Svensson, Modelling the effect of graphite morphology on the modulus of elasticity in cast irons, Int. J. Cast Met. Res. 17 (2004) 271-279. doi:10.1179/136404604225022694.

[29] L. Collini, G. Nicoletto, Determination of the relationship between microstructure and constitutive behaviour of nodular cast iron with a unit cell model, J. Strain Anal. Eng. Des. 40 (2005) 107-116. doi:10.1243/030932405X7692.

[30] ASTM International, Standard E647 - 15: Standard Test Method for Measurement of Fatigue Crack Growth Rates, (2018).

[31] K. Matouš, M.G.D. Geers, V.G. Kouznetsova, A. Gillman, A review of predictive nonlinear theories for multiscale modeling of heterogeneous materials, J. Comput. Phys. 330 (2017) 192-220. doi:10.1016/j.jcp.2016.10.070.

[32] G. Hütter, L. Zybell, M. Kuna, Micromechanisms of fracture in nodular cast iron: From experimental findings towards modeling strategies - A review, Eng. Fract. Mech. 144 (2015) 118-141. doi:10.1016/j.engfracmech.2015.06.042.

[33] A. Buljac, L. Helfen, F. Hild, T.F. Morgeneyer, Effect of void arrangement on ductile damage mechanisms in nodular graphite cast iron: In situ 3D measurements, Eng. Fract. Mech. 192 (2018) 242-261. doi:10.1016/j.engfracmech.2018.01.008.

[34] C.L. Xu, T. Andriollo, Y.B. Zhang, J.C. Hernando, J. Hattel, N. Tiedje, Micromechanical impact of solidification regions in ductile iron revealed via a 3D strain partitioning analysis method, Scr. Mater. 178 (2020) 463-467. doi:10.1016/j.scriptamat.2019.12.018.

[35] M. Meyers, K. Chawla, Mechanical Behavior of Materials, Second Edi, Cambridge University Press, Cambridge, 2009.

[36] W. Guo, Three-dimensional analyses of plastic constraint for through-thickness cracked bodies, Eng. Fract. Mech. 62 (1999) 383-407. doi:https://doi.org/10.1016/S0013-7944(98)00102-7.

[37] G.I. Barenblatt, Scaling, Self-similarity, and Intermediate Asymptotics, Cambridge University Press, Cambridge, 1996. http://ebooks.cambridge.org/ref/id/CBO9781107050242 (accessed April 26, 2016).

[38] The MathWorks, Matlab online documentation - regionprops, (2020). https://nl.mathworks.com/help/images/ref/regionprops.html. 
[39] Y. Zhang, R. Shen, M. Li, J. Pang, L. Zhang, S. Li, Z. Zhang, Mechanical damage behavior of metal matrix composites with the arbitrary morphology of particles, J. Mater. Res. Technol. 9 (2020) 7002-7012. doi:10.1016/j.jmrt.2020.05.014.

[40] W. Pantleon, Resolving the geometrically necessary dislocation content by conventional electron $\begin{array}{llllll}\text { backscattering } & \text { diffraction, } & \text { Scr. } & \text { Mater. } & 58 & \text { (2008) }\end{array}$ doi:https://doi.org/10.1016/j.scriptamat.2008.01.050. 